\title{
Requirements in designing a device for experimental investigation of threading accuracy
}

\author{
Laurențiu Slătineanu' ${ }^{1}$, Miroslav Radovanovic ${ }^{2}$, Margareta Coteață ${ }^{1, *}$, Irina Beșliu ${ }^{3}$, Oana \\ Dodun $^{1}$, Ionel Coman ${ }^{1}$, and Sergiu-Constantin Olaru ${ }^{1}$ \\ 1"Gheorghe Asachi" Technical University of Iași, Department of Machine Manufacturing \\ Technology, Blvd. D. Mangeron, 59A, Iași, Romania \\ ${ }^{2}$ University of Niš, Department of Production Engineering, Aleksandra Medvedeva 14, 18000 Niš, \\ Serbia \\ 3 "Ștefan cel Mare" University of Suceava, Department of Mechanics and Technology, Universității \\ Street, 13, Suceava, Romania
}

\begin{abstract}
When the threads are obtained by using turning tools, various factors are able to affect the machining accuracy. In order to reveal the way in which these factors exert influence on the threading accuracy, an analysis of some proper situations of obtaining threads on universal lathes and using turning tools was developed. The analysis showed that the thread profile error could be essentially affected by the position of the cutting tool active edges relative to the workpiece. Taking into consideration the results of analysis, some aspects concerning the requirements to be met during the design of a device that could be used in experimental investigation of influence exerted by some factors on the threading accuracy are presented.
\end{abstract}

\section{Introduction}

Threads are surfaces obtained by moving a certain profile along a helix curve. They are used in order to achieve a motion (threads of moving), a demountable assembly (clamping threads) or to meet special objectives (special threads). There are various criteria applicable when classifying the threads (measuring system, surface on which they are placed, the position of this surface (internal or external), direction of winding, type of profile moved along the helix curve, number of thread spirals etc.

If the problem of obtaining an external thread is stated, one could take into consideration the plastic deformation processes (rolling), the cutting processes (turning, milling, grinding), the use of distinct nonconventional machining processes etc.

The main geometrical parameters of metric external cylindrical thread are considered the average diameter, the pitch and the half of the profile angle. Some other secondary parameters could be also additionally considered: major diameter, minor diameter, the profile theoretical height, the profile real height, the angle of helicoid curve, the radius of

\footnotetext{
* Corresponding author: mcoteata@tcm.tuiasi.ro
} 
rounding profile contour etc. $[1,2,3,4]$. For each of the above mentioned parameters, adequate values for the tolerances could be established.

There are many factors able to exert influence on the thread machining accuracy: the wear of technological system components, the geometrical accuracy of the technological system components, the elastic deformation of the technological system components under the action of mechanical forces generated by the machining process, the positioning of the tool point relative to the workpiece surface etc.

Two of the thread profile errors that are analyzed when the problem of thread accuracy is formulated are the flank profile error and the error of the so-called pitch diameter. There are some groups of factors able the affect the sizes of the above mentioned two errors:

- the position of the tool cutting edges relative to the axial plane of workpiece;

- the angular position of the turning tool axis;

- the errors of the cutting tool profile etc.

Distinct aspects concerning the obtaining of threads on lathe and the ways of analyzing their accuracy constituted preoccupation subjects for the researchers interested by the problem of the manufacturing threaded parts.

Thus, Khoshdarregi and Altintas investigated the multi-point thread turning operation [3]. They considered that the threads accuracy and surface quality are crucial aspects that must be considered in order to avoid the fatigue failure and shearing of threads flanks. A generalized and systemic approach was proposed to predict the cutting forces in turning threads with multi-point inserts.

Stanciu developed an ample research aiming to highlight the influence exerted by some technological factors (tool profile errors, machine tool geometrical errors, elastic deformation of the technological system components etc.) on the dimensional and profile accuracy of threads obtained on lathes [5]. Some problems concerning the machining accuracy in the case of threaded surfaces of screws gears obtained by thread whirling were investigated by G. Crețu [2], by taking into consideration the influence exerted by the cutting conditions and geometrical parameters of cutting tool on the accuracy of machined surface. Machining errors appeared during the thread whirling process were also investigated by V.V. Merticaru, who addressed the problem of modeling the influence exerted by the cutting tools accuracy and setting the position of cutting tools on the machining accuracy [4].

In this paper, the authors took into consideration the analysis of the possibility to design a device for experimental study of influence exerted by the position of the turning tool active edges on the thread geometrical and profile errors.

\section{Hypotheses concerning the influence of various factors on the thread profile accuracy obtained by using a turning tool}

In Figure 1, a schematic presentation of the external metric thread profile and some of main its characteristics are presented. The main geometrical characteristics are the nominal (basic) diameter, major and minor diameter, the pitch, the angle of thread flank inclination. If the thread profile is examined, one could notice that this profile has essentially the shape of an equilateral triangle; a rounded zone is placed at the bottom of the thread open space. In such a case, in the axial section of the threaded cylindrical part, the thread flank is represented by a line segment.

In the case of the threaded surface, one of the machining accuracy indicators could be the error relative to the given profile and the error relative to the given shape of the surface, respectively. The error to the given shape of the profile is defined as the maximum distance between the real profile and the adjacent profile of given shape, this distance being measured between the limits of the reference length. 


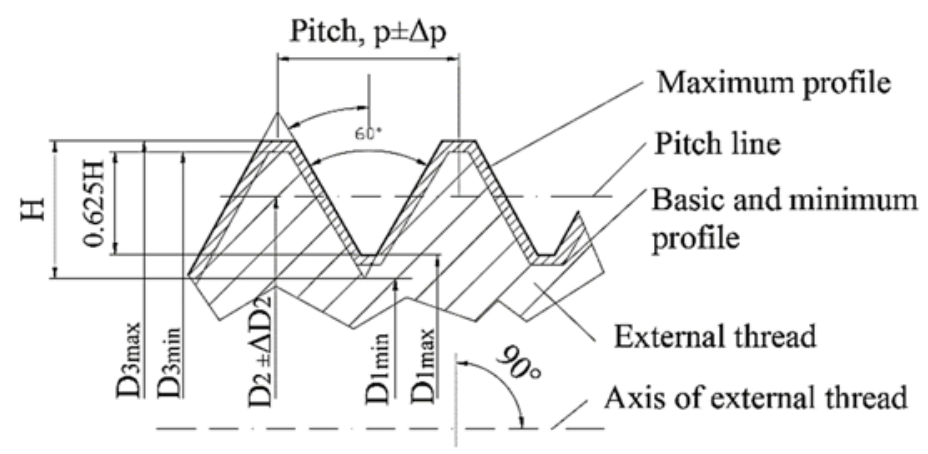

Fig. 1. Characteristics of metric external thread.

On the other hand, the error relative to the given shape of the surface could be defined as the maximum distance between the real surface and the adjacent surface of given shape, between the limits of the reference length.

As above mentioned, a common way of obtaining external threads is based on a machining process developed on a lathe. In such a case, the threaded surface is gradually generated using an apron longitudinal movement strictly correlated with the main shaft rotation. It is known that in the case of universal lathes, the position of the cutting tool in the tool holder is usually established by the lathe operator; in the case of a computer

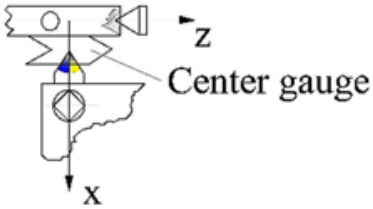

Fig. 2. Positioning the threading tool on universal lathe by means of a center gauge. numerical controlled lathe, the position of the threading tool is established out of the machine tool, using a device for pre-setting the position of threaded tool.

When a metric threaded surface is obtained on a universal lathe, in order to correctly position the tool body in tool holder relative to the workpiece axis before starting the threading process, a center gauge could be used (Figure 2). One could mention that even in such a case, however there are various factors deriving from the position of the tool edges able to affect the profile error of the machined thread. Some such situations could be examined.

In a coordinate system $x O y z$ (Figure 3), the correctness of thread profile could be determined essentially by the position of the threading tool along the $O y$ axis, and by the possible rotations of the tool around the axes $O x, O y$ and $O z$. One could mention that a tool positioning error along the $O z$ axis and respectively along the $O y$ axis practically does not affect the thread profile error, but they could exert influence on the sizes of other characteristics corresponding to threaded surface.

In the case of a tool lathe characterized by a value equal to zero of the side rake angle, the two tool active edges are placed in the workpiece axial plane. Due to the fact that the rectilinear line segment corresponding to one tool active edge intersects the workpiece axis, if there is not the threading tool longitudinal

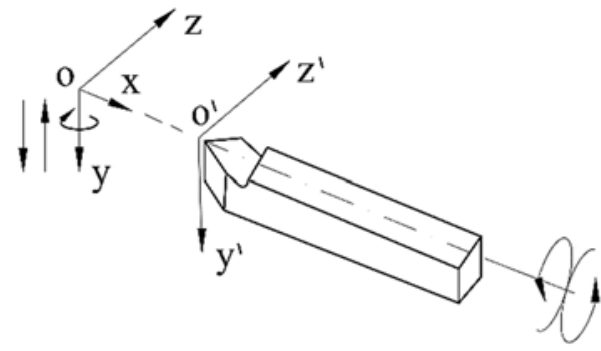

Fig. 3. Main positioning errors of the threading tool. 


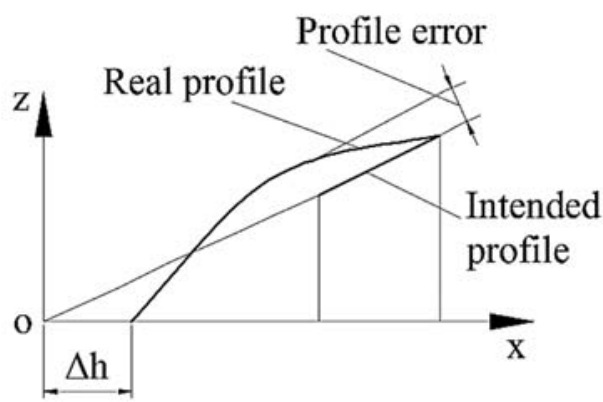

Fig. 4. Error generated by placing the threading tool over or under the horizontal plane of the main shaft rotation axis. movement, a conical surface could be generated during the transversal feed motion.

If there is only a wrong threading tool position along the $O y$ axis (this meaning that the tool corner is placed over or under the horizontal plane of the workpiece axis), the threading tool rectilinear edge will not intersect the workpiece axis and a hyperbolic surface will be generated (Figure 4). The minimum distance $\Delta h$ between the line corresponding to the threading tool cutting edge and the lathe main shaft rotation axis will correspond to the distance between the tool corner and the horizontal plane of the main shaft rotation axis (Figure 4).

If in the $x O y z$ coordinate system origin $O$ is placed on the workpiece axis (Figure 4) and the threading tool has a position rotated relative to the $O z$ axis, practically profile errors will not appear; but one could mention that in such a situation, an error of the thread basic and minor diameters could be generated. From theoretical point of view, the major diameter of the external threaded surface could be determined by the diameter of the initial cylindrical surface corresponding to the workpiece. Practically, due to a process of plastic deformation of workpiece material, a certain increase of the threaded surface major diameter could be observed.

If the origin $O^{\prime}$ of the coordinate system is placed in a position corresponding to the bottom of the future threaded surface and the threading tool is rotated around the new $\mathrm{Oz}$ ' axis (Figure 3), the threading tool active edge does not intersect the workpiece rotation axis and a hyperbolic surface is also generated.

If the threading tool has a position rotated relative to the $O x$ axis, one could notice that the rectilinear line segment corresponding to one of the threading tool active edges also does not intersect the workpiece axis and a hyperbolic surface could be generated as a consequence of a transversal movement achieved by the threading tool and when there is not an apron longitudinal movement.

It is known that during the process of setting the position of threading tool, some errors could affect each of the above mentioned aspects of positioning the threading tool.

In all this cases, an experimental research could show the influence exerted by the positioning errors corresponding to the threading tool and their weight in the total positioning error, but a theoretical appreciation of these errors could be also useful.

In the case of the threading tools whose cutting edges are not placed in the plane which contains the rotation axis of lathe main shaft, the two cutting edges do not intersect the rotation axis $\mathrm{Oz}$ and, when there is not the apron longitudinal movement, a

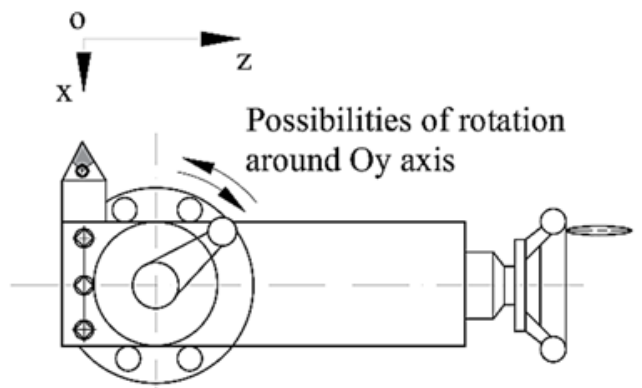
hyperbolic surface could be

Fig. 5. Rotation of threading tool around the Oy axis ensured by the rotation of universal lathe usual tool holder. 


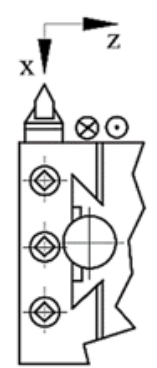

Fig. 6. Tool holder for positioning the threading tool along the $\mathrm{Oy}$ axis. generated instead of a conical one. Taking into consideration this situation, an estimated theoretical evaluation of the machined thread shape error could be developed, by determining the distance from the hyperbolic surface to the conical surface, in the space interval corresponding to the threaded surface of interest (fig. 4).

One of the frequent errors able to affect the thread parameters is generated by the position of the turning tool.

\section{Problems and solutions in the case of a device for experimental investigation of the profile error when using a turning tool}

In order to conceive and materialize a device for experimental research of the profile errors corresponding to an external metric thread in accordance with the above mentioned aspects, some general requests could be addressed.

As essential requests concerning this device, one could take into consideration the possibilities of changing the position of the threading tool along the $O y$ axis and of rotating the threading tool around the axes $O x, O y, O z$, respectively.

If the conditions offered by a turning process developed on a universal lathe are analyzed, one could notice that the rotation around $O y$ axis could be relatively easily ensured by rotating the slide of the tool holder around the vertical axis (Figure 5). Usually, this operation needs the unscrewing some nuts and the rotation of the guide slide following the angular indication existing on the apron. In this way, one could notice that the searched device could ensure the positioning of the threading tool along the $O y$ axis and the two rotations around the axis $O z$ and $O x$, respectively.

It is known that usually, to position the turning tool so that the tool corner is placed in the horizontal plane of the main shaft rotation axis, some calibrated blades could be placed under the turning tool up to obtaining the desired position of the turning tool. The operation is sometimes achieved by using the position of the live center corner placed in the conical hole from the

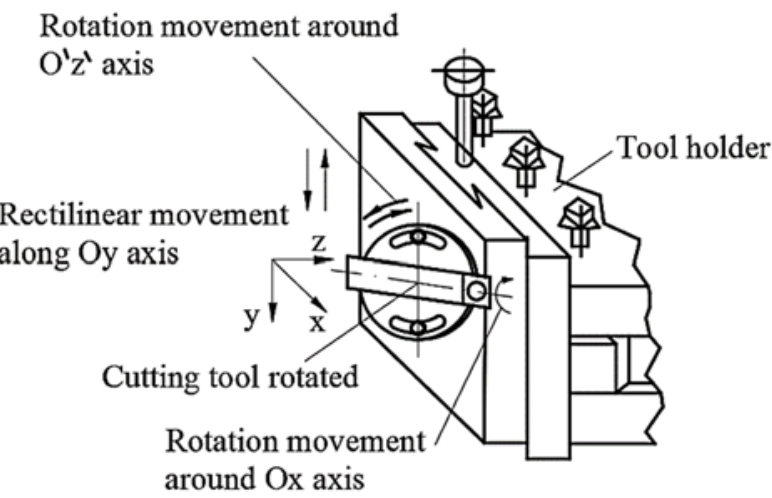

Fig. 7. Schematic representation of the device for the experimental study of influence exerted by some positioning errors of the threading tool on the thread profile. tailstock. This solution could be also applied in the case of the device for investigation the influence exerted by the position of the threading tool on the machined thread accuracy.

An easier possibility to position the threading tool along the $O y$ axis could include a slide able to be moved and fixed in the desired position, eventually using a measuring scale drawn on the slide or on its guide (Figure 6 and Figure 7).

The rotation of the threading tool around an axis parallel to the $O z$ axis could not be achieved by the common solutions existing on the universal lathe and an adequate 
constructive solution could be included in the device able to facilitate the investigation of the thread profile errors (Figure 7).

The third rotation of the treading tool that around the $O x$ axis could be inspired by the solution of tools holder necessary in the case of tools used on drilling and milling machines and such a solution could be applied in the case of the device for investigating the machining errors generated by the position of the threading tool when using a universal lathe.

Thus, the main requirements for a device utilizable to experimentally evaluate the influence exerted by the threading tool position on the profile errors of the threaded surfaces, the following ways of changing the position of the threading tool have to be ensured:

- $\quad$ positioning along the $O y$ axis;

- $\quad$ rotations around the axes $O x, O y, O z$.

\section{Conclusions}

In principle, cylindrical threaded surfaces are achieved by moving a certain profile along a cylindrical helix. If this profile is an equilateral triangle, a so-called metric thread could be obtained. On the accuracy of the threaded surface, many factors could exert influence. If only the position of the threading tool is considered, one could notice that the profile error of threaded surface could be affected by the position of the threading tool cutting edges relative to the workpiece. In order to highlight the influence exerted by the threading tool position on the threaded surfaces accuracy, an analysis of some specific positions of the threading tool were considered. This analysis showed that the position of the threading tool along $O y$ axis and respectively the rotation of the threading tool around the axes $O x, O y$ and $\mathrm{Oz}$ or around lines parallel relative to these axes could affect the profile accuracy of the threaded surface. The rotation of the threading tool around $O y$ axis could be easily achieved by rotating the lathe common tool holder. In order to materialize the threading tool positioning along the $O y$ axis and the rotation of the threading tool around $O z$ and $O x$ axes, a device that could be clamped in the lathe common tool holder was conceived. In the future, there is the intention to achieve a device that could be used to experimentally evaluate the influence of the threading tool position on the profile errors of the threaded surfaces.

\section{References}

1. Advanced Mechanical Engineering Solutions. Unified screw threads and tolerances calculator (inch), available: http://www.amesweb.info/Screws/AsmeUnifiedInchScrew Thread.aspx, accessed: 25.02.2017 (2013)

2. G. Crețu, Theoretical and practical contributions in the field of gear screws using the principle of thread whirling. Doctoral thesis (in Romanian) (Iași, Universitatea Tehnică "Gheorghe Asachi”, 1997)

3. M.R. Khoshdarregi, Y. Altintas, Generalized modeling of chip geometry and cutting forces in multi-point thread turning, Int. J. Mach. Tools Manuf. 98 (2015)

4. V.V. Merticaru, Theoretical and experimental researches concerning the accuracy at thread whirling. Doctoral thesis (in Romanian) (Iași, Universitatea Tehnică "Gh. Asachi”, 2003) 
5. A.R. Stanciu, Contributions to the study of accuracy corresponding to threads obtained by turning tools and multipoint turning tools. Doctoral thesis ("Gheorghe Asachi” Technical University of Iași, Romania, 2008) 